\title{
Diverse cellular architecture of atherosclerotic plaque derives from clonal expansion of a few medial SMCs
}

\author{
Kevin Jacobsen, ${ }^{1,2}$ Marie Bek Lund, ${ }^{1}$ Jeong Shim, ${ }^{1}$ Stine Gunnersen, ${ }^{1}$ Ernst-Martin Füchtbauer, ${ }^{3}$ \\ Mads Kjolby, ${ }^{4}$ Laura Carramolino, ${ }^{2}$ and Jacob Fog Bentzon ${ }^{1,2}$ \\ 'Deparment of Clinical Medicine, Aarhus University, Aarhus, Denmark. ${ }^{2}$ Centro Nacional de Investigaciones \\ Cardiovasculares Carlos III (CNIC), Madrid, Spain. ${ }^{3}$ Department of Molecular Biology and Genetics and ${ }^{4}$ Department of \\ Biomedicine, Aarhus University, Aarhus, Denmark.
}

\begin{abstract}
Fibrous cap smooth muscle cells (SMCs) protect atherosclerotic lesions from rupturing and causing thrombosis, while other plaque SMCs may have detrimental roles in plaque development. To gain insight into recruitment of different plaque SMCs, we mapped their clonal architecture in aggregation chimeras of $e \mathrm{GFP}^{+} \mathrm{Apoe}^{-/-}$and $\mathrm{Apoe}^{-/-}$mouse embryos and in mice with a mosaic expression of fluorescent proteins in medial SMCs that were rendered atherosclerotic by PCSK9induced hypercholesterolemia. Fibrous caps in aggregation chimeras were found constructed from large, endothelial-aligned layers of either $\mathrm{eGFP}^{+}$or nonfluorescent SMCs, indicating substantial clonal expansion of a few cells. Similarly, plaques in mice with SMC-restricted Confetti expression showed oligoclonal SMC populations with little intermixing between the progeny of different medial SMCs. Phenotypes comprised both ACTA2+ SMCs in the cap and heterogeneous ACTA2 SMCs in the plaque interior, including chondrocyte-like cells and cells with intracellular lipid and crystalline material. Fibrous cap SMCs were invariably arranged in endothelium-aligned clonal sheets, confirming results in the aggregation chimeras. Analysis of the clonal structure showed that a low number of local medial SMCs partake in atherosclerosis and that single medial SMCs can produce several different SMC phenotypes in plaque. The combined results show that few medial SMCs proliferate to form the entire phenotypically heterogeneous plaque SMC population in murine atherosclerosis.
\end{abstract}

Conflict of interest: The authors have declared that no conflict of interest exists.

Submitted: June 23, 2017

Accepted: August 31, 2017

Published: October 5, 2017

Reference information: JCI Insight. 2017;2(19):e95890. https://doi.org/10.1172/jici. insight.95890.

\section{Introduction}

Atherosclerotic plaque smooth muscle cells (SMCs) have traditionally been identified in atherosclerotic plaques by detection of contractile myofilaments or the proteins contained herein (e.g., smooth muscle $\alpha$-actin [ACTA2] and smooth muscle myosin heavy chain [MYH11]). Such contractile SMCs reside predominantly in the superficial parts of lesions and, together with the fibrous tissue matrix they produce, make up the fibrous cap. Recent studies tracking the fate of arterial SMCs in murine and human atherosclerosis have shown that plaques also contain sizeable populations of SMCs without contractile proteins (1-3). The phenotypes and functional repertoire of these modulated SMCs are still not well delineated.

Thin fibrous caps render plaques vulnerable to rupture and are the underlying cause of $60 \%-80 \%$ of lethal coronary thrombi $(4,5)$. Previous research has implicated replicative exhaustion and resultant loss of cap SMCs as a contributing cause to cap degradation. Human fibrous cap SMCs bear markers of cellular senescence, have reduced rates of mitosis, and have shorter telomeres than SMCs of the neighboring normal vessel wall (6). In mice, protecting SMCs against replicative senescence increases cap thickness (7). Replicative senescence could result from massive clonal expansion of a few SMCs during cap formation or from continued turnover of a multiclonal population with a high rate of cell death. Even though the observed rates of mitosis and cell death in plaques at any point in time are low (8), the protracted history of human fibroatheroma development allows for both possibilities.

Previous attempts to address this question in human arterial specimens have been inconclusive. Analyses of X chromosome inactivation in homogenized microdissected arteries have indicated that ACTA2 ${ }^{+}$ SMC-rich regions, including fibrous cap SMCs, contain large clonal populations (9). These analyses, 
however, could not resolve whether the apparent clonal SMC expansion occurred during embryonic development, body growth, or atherogenesis, with the problem being that the normal arterial intima and media - from where plaque SMCs were recruited - also appeared to consist of large coherent populations of SMCs derived from single embryonic founder cells $(9,10)$.

In the present study, we use experimental atherosclerosis models to gain insight into the development and clonal architecture of the fibrous cap, as well as the rest of the SMC population. We show that the normal murine aorta has a small SMC patch size allowing for investigation of clonal SMC expansion in aggregation chimeras, and we use chimeras and mice with a randomly recombining fluorescent transgene to show that plaque SMCs, including fibrous cap, chondrocyte-like, and lipid-filled SMCs, undergo significant clonal expansion during plaque development. Furthermore, we show that the fibrous cap population has a sheet-like, endothelium-aligned architecture indicative of an endothelium-cued recruitment process.

\section{Results}

Clonal analysis in chimeric Apoe ${ }^{-1-}$ mice. We examined clonal relationships of ACTA2 ${ }^{+}$fibrous cap SMCs in mouse chimeras created by aggregating 8-cell embryos from matings of $e G F P^{+} A p o e^{-/-}$and Apoe $e^{-/-}$mice ( $n$ = 9) (Figure 1A). The mice were kept on chow until 10 months to develop atherosclerosis. By initial analysis of the myocardium, 4 mice were found to be balanced $e G F P^{+} A p o e^{-/-} \leftrightarrow A p o e^{-/-}$chimeras. The others had either no $(n=3)$ or nearly ubiquitous $(n=2)$ eGFP expression in the myocardium and were likely generated by aggregation of 8-cell embryos of the same genotype $\left(A p o e^{-/-} \leftrightarrow A p o e^{-{ }_{-}}\right.$and $e G F P^{+} A p o e^{-I_{-}} \leftrightarrow$ eGFP Apoe $\left.^{--}\right)$.

The clonal architecture of the aorta has not been previously reported for mice. Analysis of ACTA2 SMCs in the aortic media of aggregation chimeras showed that the size of patches, defined as groups of contiguous cells of the same color, was small, with cells of the less common genotype interspersed between the others in groups comprising only a few cell profiles. This was the case in both the cross-sectional (aortic root) and in the longitudinal (aortic arch) direction (Figure 1, B and C). In the positive control $e G F P^{+} A_{p o e^{-1}}$ $\leftrightarrow e G F P^{+} \mathrm{Apoe}^{-/-}$mice, all medial SMCs expressed eGFP, albeit with some heterogeneity in expression levels (Supplemental Figure 1; supplemental material available online with this article; https://doi.org/10.1172/ jci.insight.95890DS1).

The clonal pattern of the murine aortic media is consistent with the developmental processes described for the murine pulmonary artery, where intermixing of cells occurs during recruitment and differentiation of SMCs along the nascent endothelium (11). Curiously, this is very different from the pattern reported for human aortas and coronary arteries, proposed to have large coherent patches of SMCs deriving from the same embryonic ancestor cells $(9,10)$. Importantly, the small patch size of $\mathrm{eGFP}^{+}$and nonfluorescent SMCs in the media allowed us to analyze for SMC clonal expansion during the formation of atherosclerotic plaques. Both in the aortic root and inner aortic arch, we found clear evidence that plaque ACTA2 fibrous cap SMCs are an oligoclonal population, consisting of coherent sheets of cells with the same genotype. The patch size of ACTA2 $2^{+}$SMCs in the plaque was considerably larger than seen in the arterial media in all of the 4 chimeras examined (Figure 1, B and C). Combined with existing evidence that medial SMCs are the precursors of $\mathrm{ACTA}^{+} \mathrm{SMCs}$ in mouse atherosclerosis (1-3), this indicates that plaque ACTA2 SMCs arise by substantial proliferation of a limited number of medial SMCs. When fibrous caps contained more than one genotype of cells, they were often seen arranged in a layered architecture.

Medial SMC fate mapping in mosaic mice. The analysis of aggregation chimeras left several questions unanswered. First, it only allowed the identification of SMCs expressing ACTA2, which has high specificity but low sensitivity for the SMC lineage in atherosclerotic plaques (1-3). Second, it did not provide direct tracing of cell origin back to medial SMCs. Third, analysis of SMC migration and proliferation in Apoe ${ }^{-/-}$mice was potentially biased by the lack of APOE, which has direct inhibitory effects on SMC migration and proliferation (12). To overcome the first 2 limitations, we turned to Myh11-CreER ${ }^{T 2}$ Confetti mice. The Myh11-CreER ${ }^{T 2}$ line has previously been shown to provide inducible CRE recombinase activity that is highly specific to SMCs with no detectable recombination in other tissues or circulating cells $(13,14)$. The Confetti transgene harbors the coding sequence of 4 fluorescent proteins that can be randomly recombined under the influence of CRE to give expression of membrane-bound CFP (mCFP), nuclear GFP (nGFP), yellow fluorescent protein (YFP), or red fluorescent protein (RFP) (15). The combination of the 2 transgenes, thus, allowed us to track the progeny of single medial SMCs during the development of atherosclerosis, irrespective of phenotypic changes they might undergo. To meet the third limitation, we induced atherosclerosis without 
A
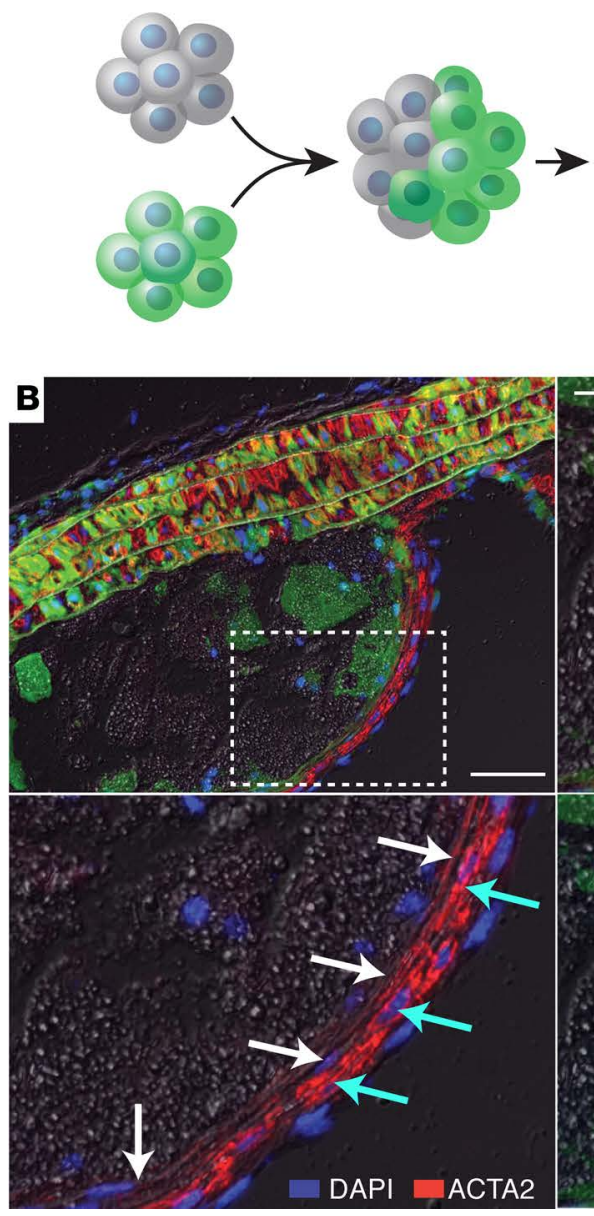
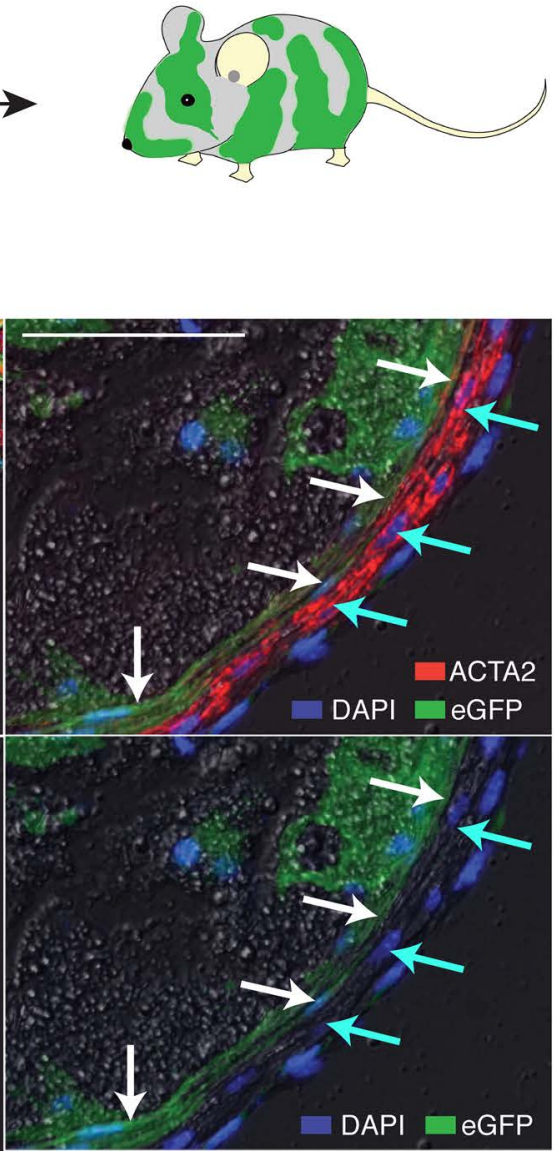

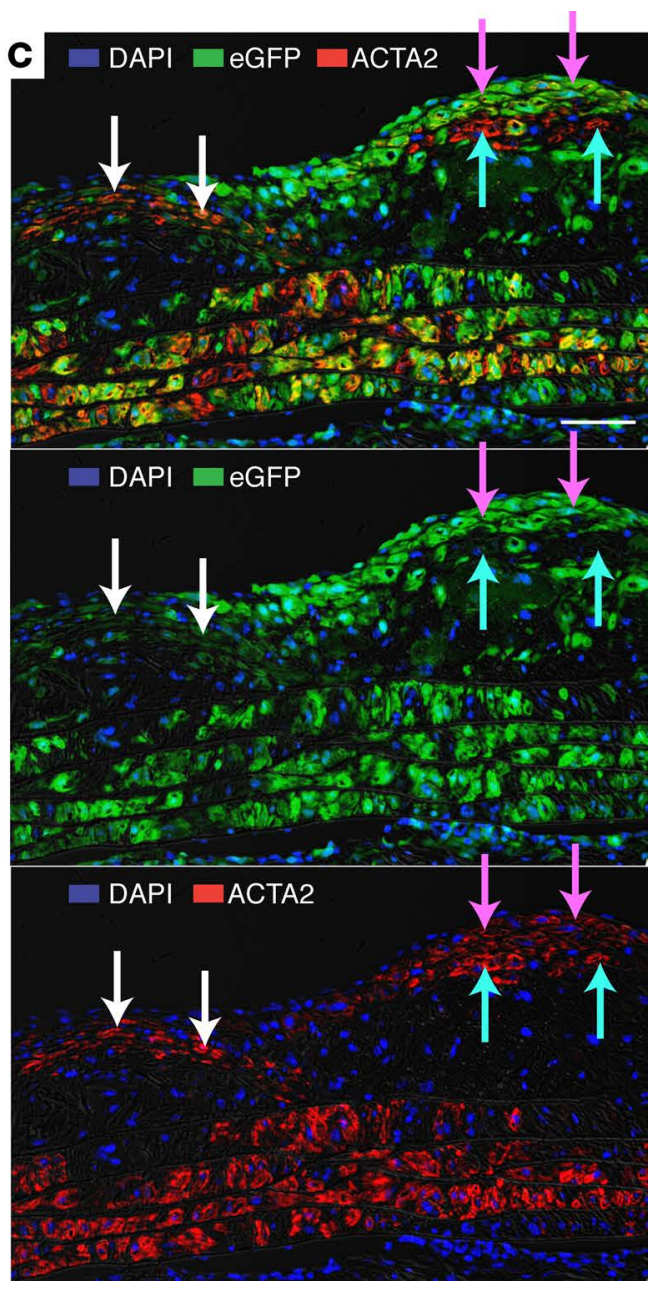

Figure 1. Plaque ACTA2 $2^{+}$SMCs are oligoclonal in Apoe $^{-/-}$aggregation chimeras. (A) Chimeras consisting of a mixture of eGFP+ and nonfluorescent cells were created by aggregating 8-cell embryos. (B) Cross-sectioned aortic root lesion showing ACTA2+ SMCs (red) organized in subendothelial layers of nonfluorescent (cyan arrows, high ACTA2 levels) and eGFP+ (green, white arrows, low ACTA2 levels). The size of the coherent plaque SMC patches are much larger than the patch size of the arterial media, indicating that substantial clonal expansion has occurred. (C) Similar findings in a longitudinal section of an aortic arch lesion. Coherent groups of nonfluorescent (cyan arrows) and eGFP+ (white and magenta arrows) ACTA2 ${ }^{+}$cells are present. Note that expression levels of eGFP are variable among patches of eGFP+ cells. Grayscale, differential interference contrast. Scale bars: $50 \mu \mathrm{m}$. Images are representative of the findings in 4 aggregation chimeras.

deleting Apoe with adeno-associated virus encoding a murine gain-of-function mutant (D377Y) of PCSK9 in hepatocytes, which renders the mice functionally LDL receptor deficient and severely hypercholesterolemic when fed a high-fat diet (16).

We induced CRE recombinase activity between 6 and 8 weeks of age in Myh11-CreER ${ }^{T 2}$ Confetti mice and in Myh11-CreER ${ }^{T 2}$ littermate controls. Three to 5 days after the last injection, when stable mosaic expression of fluorescent proteins in medial SMCs had been achieved, atherosclerosis was initiated with an injection of rAAV8-D377Y-PCSK9 and high-fat diet.

Plasma cholesterol levels and the development of atherosclerosis at 12, 24, and 36 weeks after the recombinant adeno-associated virus (rAAV) injection are shown in Figure 2, A and B. The total cholesterol levels achieved were lower and the variation higher than previously reported by us for the rAAV8-D377Y-PCSK9 technique (16). This may have been due to the close temporal proximity of the liver-directed rAAV8-D377YPCSK9 gene transfer and the series of hepatotoxic tamoxifen injections, since other work conducted by us concurrently (unpublished observations, 2015), in which rAAV8-D377Y-PCSK9 treatment was deferred 1 month after the last tamoxifen injection, led to levels and variation of cholesterol similar to our previous report (16).

No significant effects of the Confetti transgene on plasma cholesterol levels were noted, but Myh11CreER ${ }^{T 2}$ Confetti mice developed significantly less atherosclerosis compared with similarly treated Myh11-CreER ${ }^{T 2}$ littermates (Figure 2B). 
A

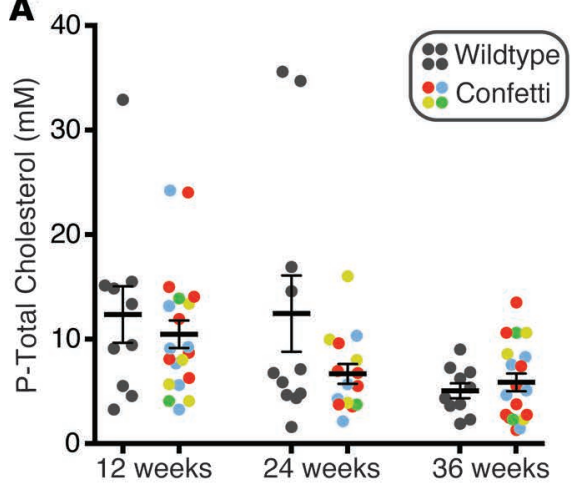

C

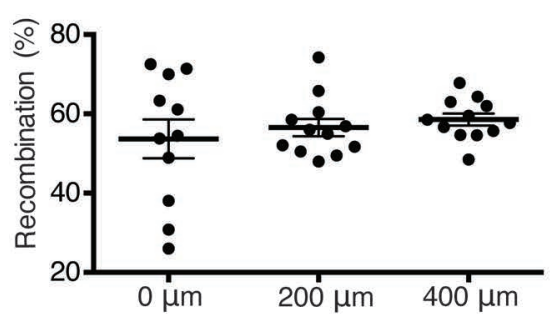

B

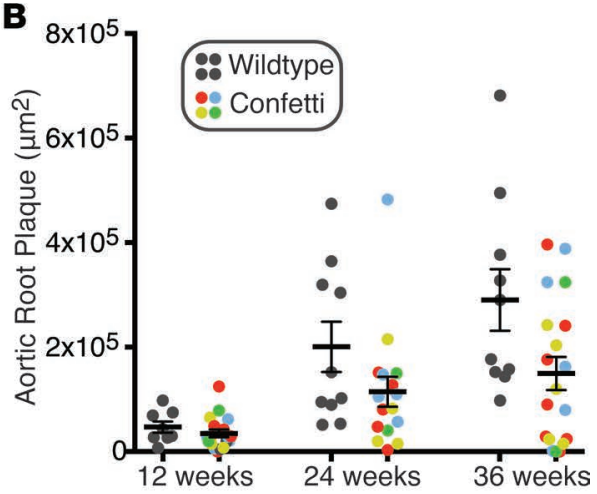

D

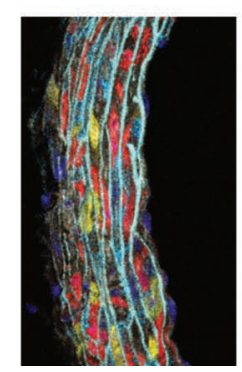

Figure 2. Induction of hypercholesterolemia and atherosclerosis in mosaic mice. (A) Plasma total cholesterol measured at 12, 24, and 36 weeks after rAAV8-D377Y-PCSK9 injection. (B) Atherosclerosis measured at the level of the commissures of the aortic valves revealing a retarding effect of the Confetti transgene on atherogenesis. $P=0.004$ for difference between genotypes (2-way ANOVA). $n=8-10$ and $n=15-20$ at each time point for WT and Confetti mice, respectively. (C) Recombination rate determined among nucleated medial SMC profiles at 3 levels of the aortic root in 12 Confetti mice starting at the commissures of the aortic valves. (D) Dot plot showing the average percentage-wise distribution of fluorescence colors in the mosaic mice. An example of an analyzed arterial media is shown on the left.

Recombination efficiencies, determined in undiseased segments in serial sections of the aortic root at 12 weeks of age, were $56.3 \% \pm 10.4 \%$ (mean \pm SD) (Figure $2 \mathrm{C}$ ). The most common outcome of recombination was expression of RFP, followed by YFP and mCFP, whereas nGFP expression occurred infrequently (Figure 2D). Our observations are consistent with the reported lower frequency of recombination events involving the LoxP site preceding the nGFP cassette (https://www.jax.org/strain/017492). The less than complete recombination aids clonal analysis by lowering the chance that the progeny of different medial SMCs of the same color will occupy the same region of plaque. This is particularly the case for mCFP, which is present in only $9.0 \%$ of medial SMCs, whereas RFP with a frequency in medial SMCs of $30.5 \%$ should be interpreted more carefully, as discussed further below, because of the higher chance that 2 independent SMCs moving into atherosclerosis could have the same color. Consistent with previous reports on Myh11-CreER ${ }^{T 2}$ mice $(13,14)$, we did not detect recombination, except in the arterial media of the aorta and neighboring small arteries and arterioles.

Recruitment of plaque SMCs. To focus our clonal analysis on progressed lesions containing SMCs, rather than early foam cell lesions, we selected the 10 mice with most atherosclerosis at 12, 24, and 36 weeks for further analysis. In each, we examined serial sections, spaced at $200 \mu \mathrm{m}$ and covering the entire aortic root plaques. Figure 3 , $\mathrm{A}$ and $\mathrm{B}$, depicts lesion volume and cellularity in each coronary sinus; they are analyzed individually, since they represent separate atherogenic processes. Plaque development was much faster in the left and noncoronary than the right coronary sinus. A decline in cellularity (nuclei per $\mathrm{mm}^{2}$ in serial sections) was notable over time, which — unlike plaque volume - showed no differences between sini (Figure 3B). Recruitment of fluorescent plaque SMCs followed the pattern of plaque growth with a wave of SMC recruitment in the left and noncoronary sinus between 12 and 24 weeks of plaque development, but this was not accomplished until after 24 weeks in the right coronary sinus (Figure 3C). Overall, our analysis encompassed approximately 2,500 plaque SMCs.

Clonal architecture of plaque SMCs. The pattern of fluorescent protein expression in plaque SMCs was substantially different from the single-cell mosaic configuration of the arterial media. Rather than being individual cells migrating into plaques from the media, the vast majority of SMCs belonged to large same-color cell patches occupying separate parts of the plaque (Figure 4).

Figure 5A illustrates the organization of the fluorescent plaque SMC population at each time point. We found 133 SMC patches (1.5\% nGFP, 17.3\% mCFP, 35.3\% YFP, 45.9\% RFP). In 6 cases, 2 patches of different colors were overlapped, giving an area with a mixture of SMCs of 2 colors (Figure 4D). The distribution of patch colors was not different from the distribution of colors among medial SMCs $\left(\chi^{2}\right.$ test, $\left.P=0.14\right)$.

The number of SMC patches increased from 12-24 weeks but was not significantly different between 24 and 36 weeks of plaque development (Figure 5B). There was a trend toward larger patch size at 36 weeks 
A

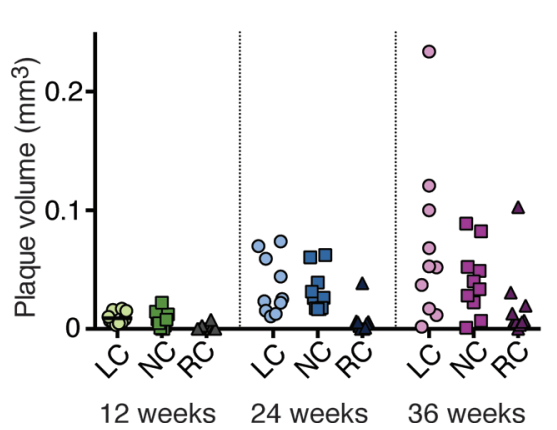

B

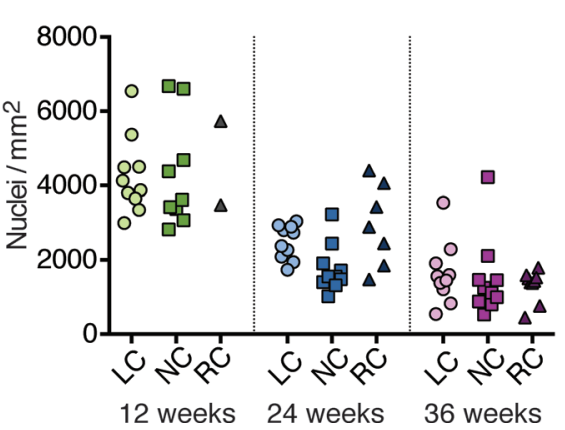

C

SMC recruitment

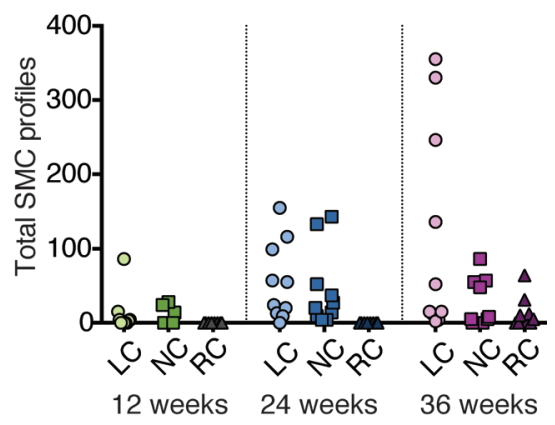

Figure 3. SMC recruitment. (A and B) Progression and cellularity of atherosclerotic lesions in each aortic sinus at the indicated time points after induction of hypercholesterolemia. Despite the lower plaque volume in the right coronary sinus, cellularity was not different from the other sini. (C) Total number of fluorescent SMC profiles identified in serial sections through each lesion. LC, left coronary sinus. NC, noncoronary sinus. RC, right coronary sinus. $n=10$ mice at each time point.

compared with 24 weeks and 12 weeks, but the difference was not statistically significant $(P=0.06,1$-way ANOVA, log-transformed data) (Figure 5C).

The number of fluorescent SMC patches divided by the average percentage of fluorescent cells in the media (56.3\%) is a crude estimate of the number of medial SMCs that contribute to the plaque SMC population in the aortic root. For the 12-, 24-, and 36-week time points, this number was $2.1 \pm 2.4,10.2 \pm 5.3$, and 11.6 $\pm 7.3 \mathrm{SMCs}$, respectively. Importantly, these numbers could be both over- and underestimations of true values. They would be overestimations if progeny of single founder cells in some cases had split up and given rise to more than one patch, and they would be underestimations if SMC patches were sometimes derived from several founder cells.

Since only $9.0 \%$ of medial SMCs were mCFP, it is unlikely that coherent mCFP collections originated, except in rare instances, from several founder cells, considering that 2 medial SMCs recruited to the same region of plaque would be both $\mathrm{mCFP}$ in only $<1 \%$ of cases. Moreover, since patch size was not significantly different among different colors (1-way ANOVA, log-transformed, $P=0.13$ ), the other-colored patches were also consistent with such single-cell origin. Having said that, inspection of the distribution of patch sizes of each color suggested that the largest $\mathrm{RFP}^{+}$patches were indeed outliers and likely the progeny of more than one RFP founder cell (Supplemental Figure 2). This was consistent with the higher percentage of RFP $\mathrm{P}^{+}$ cells in the media (30.5\%) and, thus, the higher chance of 2 or 3 neighboring SMC clonal populations being RFP (9.3\% and 2.8\%, respectively). However, this would not have a significant impact on our estimates. Interpreting the largest $4 \mathrm{RFP}$ patches (all found at 36 weeks) as the result of 2 founders each increased the estimate for 36 weeks to $12.3 \pm 8.3$ SMCs. Furthermore, although our serial sampling density at every 200 $\mu \mathrm{m}$ allowed us to miss single or small groups of SMCs, we would be unlikely to miss larger groups. The combined data, thus, showed that not only ACTA2 $2^{+}$cap SMCs, but all plaque cells of SMC lineage, are derived from a very limited number of medial SMCs undergoing coherent oligoclonal proliferation.

Fibrous caps consist of sheet-like clonal populations. Plaque SMCs labeled with Confetti fluorescence markers exhibited clearly heterogenous phenotypes. Fibrous cap SMCs, defined as SMCs associated with the endothelium, were present in 24 lesions (17 mice). Consistent with our findings in Apoe $^{-1-}$ aggregation chimeras, fibrous caps were organized in sheet-like, same-colored patches, often spanning several consecutive serial sections (Figure 6, A and B). Immunostaining, performed after fluorescence microscopy, showed that these cells were ACTA2 ${ }^{+}$, whereas SMCs in the interior of lesions were ACTA2- (Figure 6C). Nonfluorescent $\mathrm{ACTA} 2^{+}$cap SMCs were also often present, consistent with the less than complete recombination of the Confetti transgene in the arterial media.

Other plaque SMC phenotypes. In addition to ACTA2+ cap SMCs, 2 types of SMC phenotypes were readily identified in the interior of lesions. Large, often rounded cells, typically negative for ACTA2 (Figure $6 \mathrm{C}$ ) and presumably similar to those that have previously been described as macrophage-like by other research groups $(2,14)$ were often abundant in lesions. Many of these cells had a foamy appearance of the cytoplasm especially recognizable in SMCs with cytoplasmic fluorescence (RFP or YFP) (Figure 7, A and B, and Figure 4A). In contrast with previous reports in $A p o e^{-/-}$mice $(2,14)$, we did not find the macrophage 

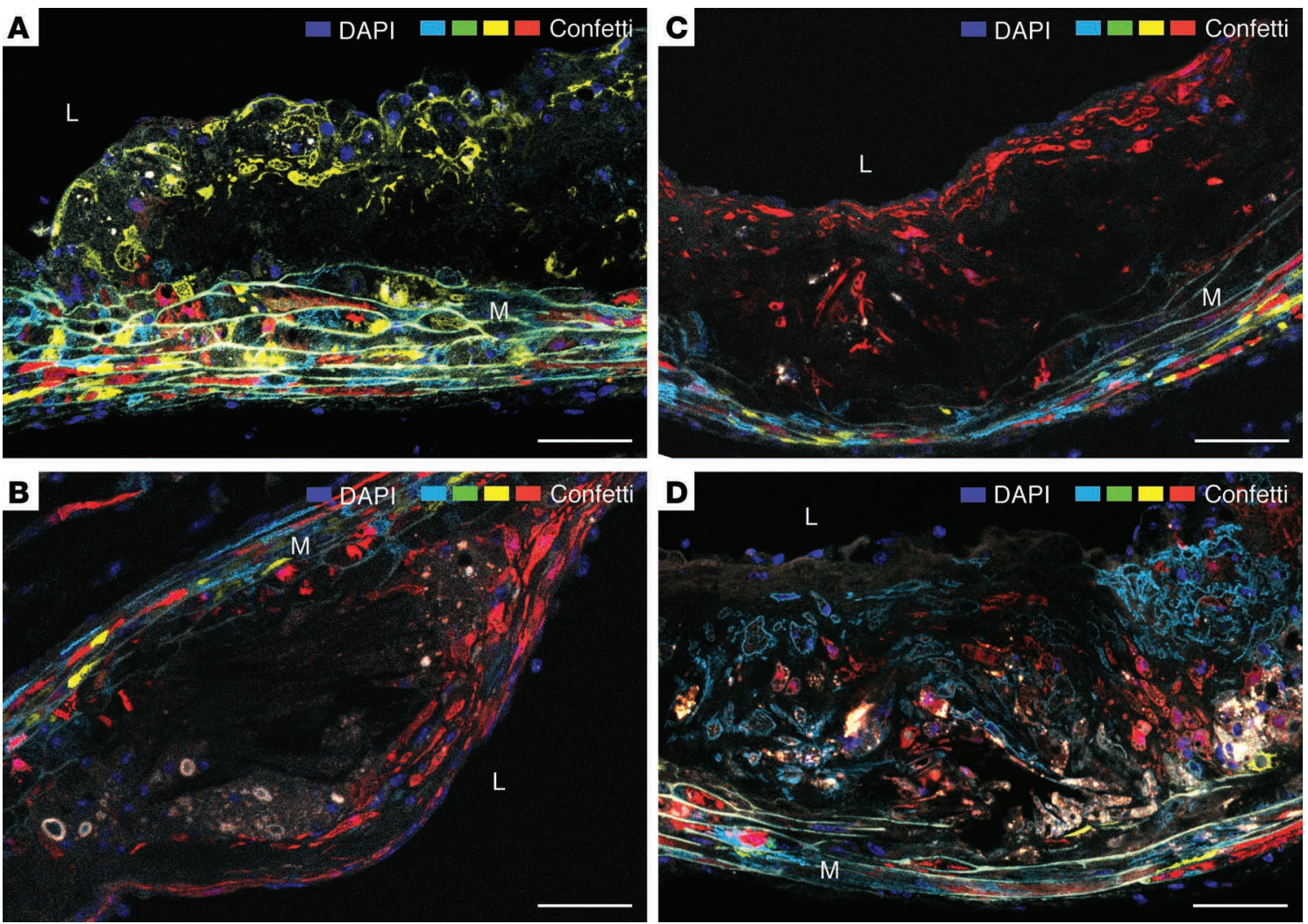

Figure 4. Plaque SMCs are organized in large clonal patches. (A-D) Four examples are shown featuring an YFP+ (A), 2 different RFP+ $S M C$ populations (B and C) at 24 weeks of plaque development, and the intermixing of 2 patches consisting of mCFP and RFP SMCs (D) at 36 weeks of plaque development. Autofluorescent material, which is variably present in the 4 lesions, has a gray/yellow color. L, lumen. M, media. Scale bars: $50 \mu \mathrm{m}$.

markers LGALS3 or CD68 expressed in SMCs (Figure 7C and Supplemental Figure 3), but laser reflection microscopy showed that foamy SMCs often contained reflective material (Figure 7D), and subsequent Oil Red O staining revealed that the majority had lipid accumulation (Figure 7, E-G).

Fluorescent chondrocyte-like cells were seen in a subset of lesions identified by their round morphology, expression of the chondrogenic transcription factor SOX9, and embedment in rich extracellular matrix (Figure 8). The identification of these cells confirms a previous lineage tracking study showing that this common plaque cell type is, at least partly, SMC derived (17) and extends it by showing that such areas of chondroid metaplasia typically represent the clonal progeny of a single medial SMC.

An important question is whether different SMC phenotypes are progeny of separate sublineages of medial SMCs. To address this, we focused on lesions that contained fluorescent SMCs of at least 2 of the 3 phenotypes described above. In 23 cases, we found that fluorescent SMCs of different phenotypes were part of a common patch (see examples in Figure 7A and Figure 8E), whereas there were only 8 lesions where different phenotypes in the plaque also belonged to differently colored patches. It is possible that some same-colored patches with 2 SMC phenotypes could be derived by 2 medial SMCs of the same color. However, their frequency very significantly exceeded the expected values under the hypothesis that 2 precursors are always needed to produce 2 different phenotypes $(P=0.0001,2$-tailed binomial test), which can be calculated from the distribution of colors in medial SMCs. Our findings were, thus, not compatible with the idea that different SMC phenotypes are always derived from different medial cells, and they show that single, differentiated medial SMCs can contribute to several different SMC phenotypes in plaques.

\section{Discussion}

Delineation of the clonal architecture of the SMC population in atherosclerosis provides important framework for understanding recruitment, fate, and function of SMCs in plaque progression and vulnerability. Here, we used aggregation chimeras and a randomly recombining transgene in 2 different atherosclerosis 
A

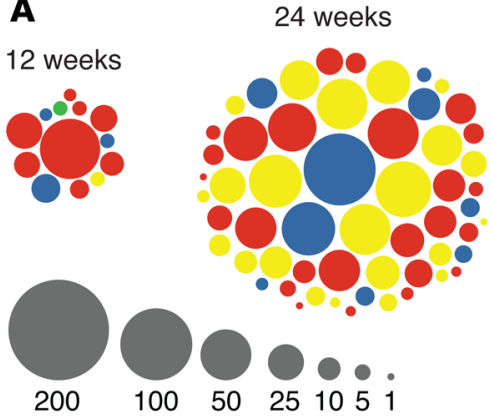

36 weeks

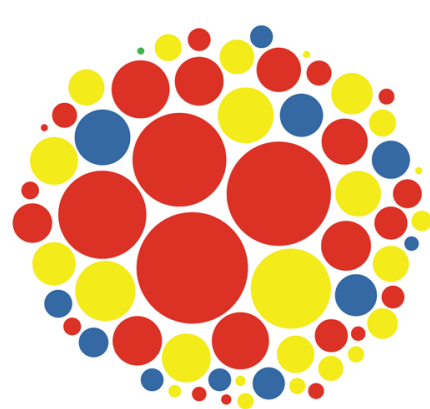

Figure 5. SMC clonal structure during plaque development. (A) Dots illustrate the size (number of cell profiles in serial sections) and color of individual SMC patches encountered in the 10 mice analyzed at each time point. (B) Number of patches in the aortic root in each mouse. The number increased from 12-24 weeks of plaque development but then remained stable (1-way ANOVA with Tukey's post-test, ${ }^{* *} P<$ 0.01). (C) Size of SMC patches quantified as number of SMC profiles in serial sections of the entire lesions. A trend toward increased patch size was seen with atherosclerosis progression, but the differences were nonsignificant (1-way ANOVA, log-transformed, $P=0.09$ ).

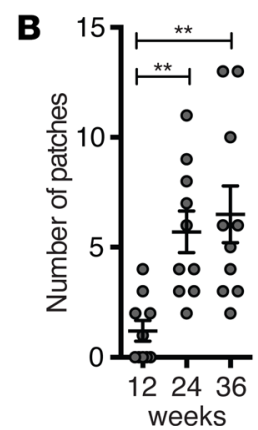

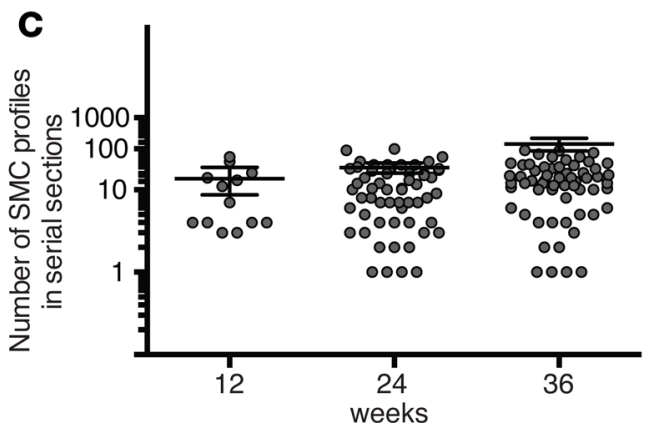

models to achieve this goal. We found that fibrous caps are built from sheets of clonally expanded progeny of single medial SMCs. As part of our approach, we also describe the patterning of the murine aortic media; we demonstrate that not only cap SMCs, but also modulated SMCs in the plaque interior, are oligoclonal cell populations derived from few medial SMCs; and we demonstrate that the plaque SMC lineage in mice includes lipid-accumulating cells.

Fibrous cap formation. The architecture of the fibrous cap, clear in both aggregation chimeras and Myh11-CreER ${ }^{T 2}$ Confetti mice, indicates an ordered process separate from the recruitment of the rest of plaque SMCs. Its often layered structure bears resemblance to the endothelium-cued development of the arterial media (18), but its clonal relationships are different. In contrast to the small patch size of the aortic media, caps were constructed by large layers of clonally expanded medial SMCs.

We speculate that the difference in the clonal structure of cap and medial SMCs, rather than reflecting different SMC recruitment mechanisms, may result from the different conditions under which the naked endothelium recruits its supporting SMC layers. In early murine atherosclerosis, the endothelium lose arterial media contact by the interpositioning of macrophage foam cells. Since the potential signals involved in SMC recruitment (e.g., PDGF signaling or direct cell contact, refs. 11, 18) can only traverse short distances, SMCs can initially only be recruited from the shoulder regions and subsequently only from progeny of the first recruited cell or cells. This is a marked difference from the development of media where the naked endothelium is surrounded by undifferentiated mesenchymal cells.

This difference may not necessarily apply to human arteries, especially at atherosclerosis-prone sites where the normal arterial intima and early atherosclerotic lesions already contain local intimal SMCs (19). These cells represent an alternative source of SMC recruitment for the cap and the rest of the plaque that is not present in mice. On the other hand, human intimal thickenings could themselves already be formed by oligoclonal expansion, leading to the same end result as described here. It will be important to revisit studies of SMC clonal relationships in humans with cellular resolution techniques to shed light on the clonal organization of arterial SMCs during artery development, intimal thickening, and plaque formation, but such studies are technically challenging.

If our findings in the mouse holds true for human atherosclerosis, one may speculate that the development of replicative senescence in cap SMCs is a direct consequence of the way that the cap is formed, rather than merely the result of continuing damage and cell replenishment alone. Unfortunately, mouse models of atherosclerosis are not well suited to address this question because cap SMCs do not undergo senescence in mice, possibly because of their extremely long telomeres (20). 

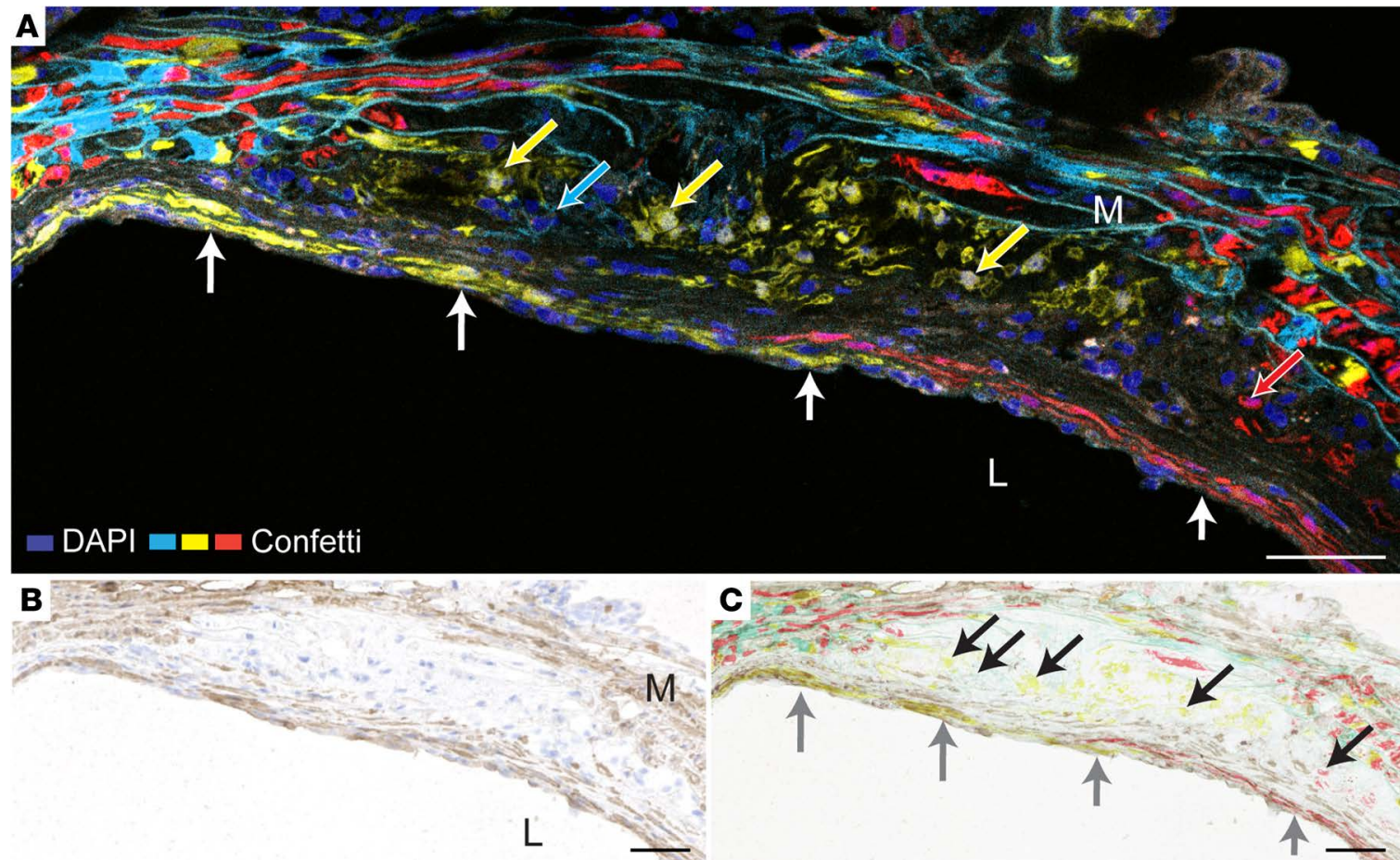

Figure 6. Fibrous caps are formed by sheets of clonally expanded medial SMCs. (A) Aortic root plaque after 24 weeks of plaque development showing a fibrous cap consisting of 2 clonally expanded layers of SMCs (YFP+ and RFP+, respectively, white arrows). SMCs of other phenotypes (YFP+, mCFP+, and $\mathrm{RFP}^{+}$, arrows in respective colors) are present in the plaque interior. This section did not contain nGFP+ SMCs. (B) The same section stained for ACTA2 (brown). (C) Overlay of fluorescence on B, showing that the fluorescent cap SMCs are ACTA2 (gray arrows). Nonfluorescent ACTA2 ${ }^{+}$cap SMCs are also present, consistent with less than complete recombination of medial SMCs. Fluorescent SMCs in the plaque interior are ACTA2- (black arrows). L, Lumen. M, Media. Scale bars: $50 \mu \mathrm{m}$.

Clonality and phenotype of non-cap SMCs. Our studies in mosaic Confetti mice allowed identification of several highly modulated SMCs in plaque interior and showed that the far majority of these cells also were part of sizeable clonal populations. This identifies SMC proliferation, rather than SMC migration, as the quantitatively dominant mechanisms underling SMC accumulation in atherosclerosis, and it distinguishes the process from other vascular pathologies, e.g., the injured rat carotid artery, where nondividing, migrating SMCs contribute sizably to neointima formation (21).

While we were finalizing our analysis, Chappell et al. reached a similar conclusion based on a comprehensive analysis of the clonal structure of SMCs in Myh11-CreER ${ }^{T 2}$ Confetti Apoe ${ }^{-1-}$ mice after 16-19 weeks of high-fat diet (22). The reported clonal structure in that experiment were, however, simpler than the one we found, with the far majority of plaques only containing SMCs of 1 or 2 Confetti colors in the setting of a higher medial SMC recombination efficiency (70\%-95\% medial SMCs) (for similar analysis of our data see Supplemental Figure 4). The reason for this difference could potentially lie in the lack of inhibitory effect of APOE on SMC proliferation in the Apoe ${ }^{-/}$background. It may also be explained by other differences between models, time points, and arterial locations. The effect of the Confetti transgene on the atherosclerotic process or the particular architecture of the fibrous cap was not analyzed directly in the paper by Chappell et al. (22). More precise estimates of the number of medial SMCs that goes into forming the plaque SMC population in different atherosclerosis models and at different time points could be obtained using mosaic mice with more recombination outcomes than the hemizygous Confetti mice used by Chappell et al. and us. Such tools have recently been published (23).

Several hypotheses can be formed for why only few out of many medial SMCs migrate and proliferate in atherosclerosis. First, the precursors for the SMC clones in plaques might be a special subgroup of medial SMCs with a cell-autonomous propensity for proliferation. The fact that the medial precursor SMCs cells are expressing MYH11, a marker of highly differentiated SMCs, speaks against a less-differentiated progenitor-like population in the media, but it is still possible that there is fixed or fluctuating heterogeneity in the phenotype of medial SMCs, allowing only a minority to partake in atherogenesis at a given point in 

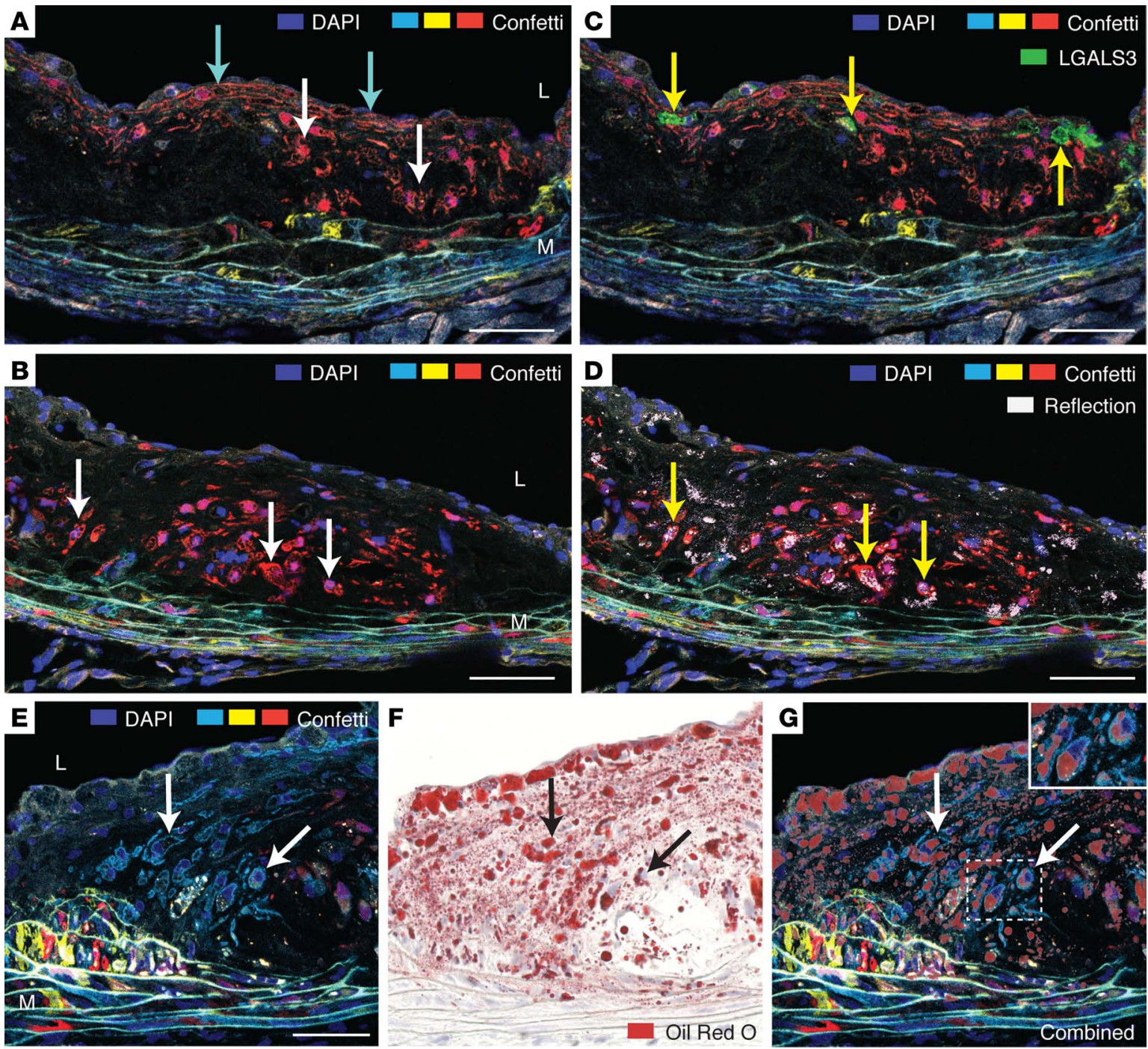

Figure 7. The plaque interior accumulates lipid-filled modulated SMCs. (A and B) Serial sections, $200 \mu \mathrm{m}$ apart, of a plaque at 24 weeks of development showing a large patch of RFP+ SMCs comprising cells with foamy cytoplasm (white arrows point to examples) and also elongated EC-associated cap SMCs (cyan arrows). (C) LGALS3+ cells are present in the plaque (yellow arrows), but no SMCs stain positive for LGALS3. LGALS3 staining was performed with a near-infrared fluorochrome but shown in green for clarity. The section did not contain nGFP+ SMCs. (D) Laser confocal reflection microscopy reveals that some of the accumulating cytoplasmatic material in modulated SMCs is reflective (yellow arrows), consistent with cholesterol crystals. (E-G) Oil Red 0 staining shows accumulation of neutral lipids in many modulated SMCs. The displayed series of images show an mCFP+ patch of SMCs (E); subsequent Oil Red $\mathrm{O}$ staining of the same section (F); and an overlay of the Oil Red $\mathrm{O}$ and fluorescent images (G). The inset shows Oil Red O-stained SMCs in higher magnification. L, Lumen. M, Media. Scale bars: $50 \mu \mathrm{m}$.

time. Second, clone precursors may have equal endogenous potential to produce plaque SMCs but may be selected by being located at the right place and the right time, e.g., where the barrier of the internal elastic lamina is breached by proteolytic enzyme activity or where a localized signal guides their expansion. Once liberated from the encaging internal elastic lamina (IEL) or modulated by the localized signal, the SMCs are free to proliferate in plaque, leaving still encaged or undisturbed neighbors behind.

Third, one may speculate that few, perhaps randomly chosen, medial SMCs that begin proliferating may actively inhibit their neighbors from taking the same fate. It is a common pattern, often orchestrated by Notch-dependent lateral inhibition signaling, that cells induced to follow a particular fate prevent surrounding cells from doing the same (24). Interestingly, Notch pathways are crucial for SMC recruitment to developing arteries $(18,25)$, and Notch elements are heavily regulated in modulated SMCs after arterial injury (26).

Different plaque SMC phenotypes. The total abundance of the SMC lineage in atherosclerotic plaques and their modulation to multiple different phenotypes suggest that they may undertake important 

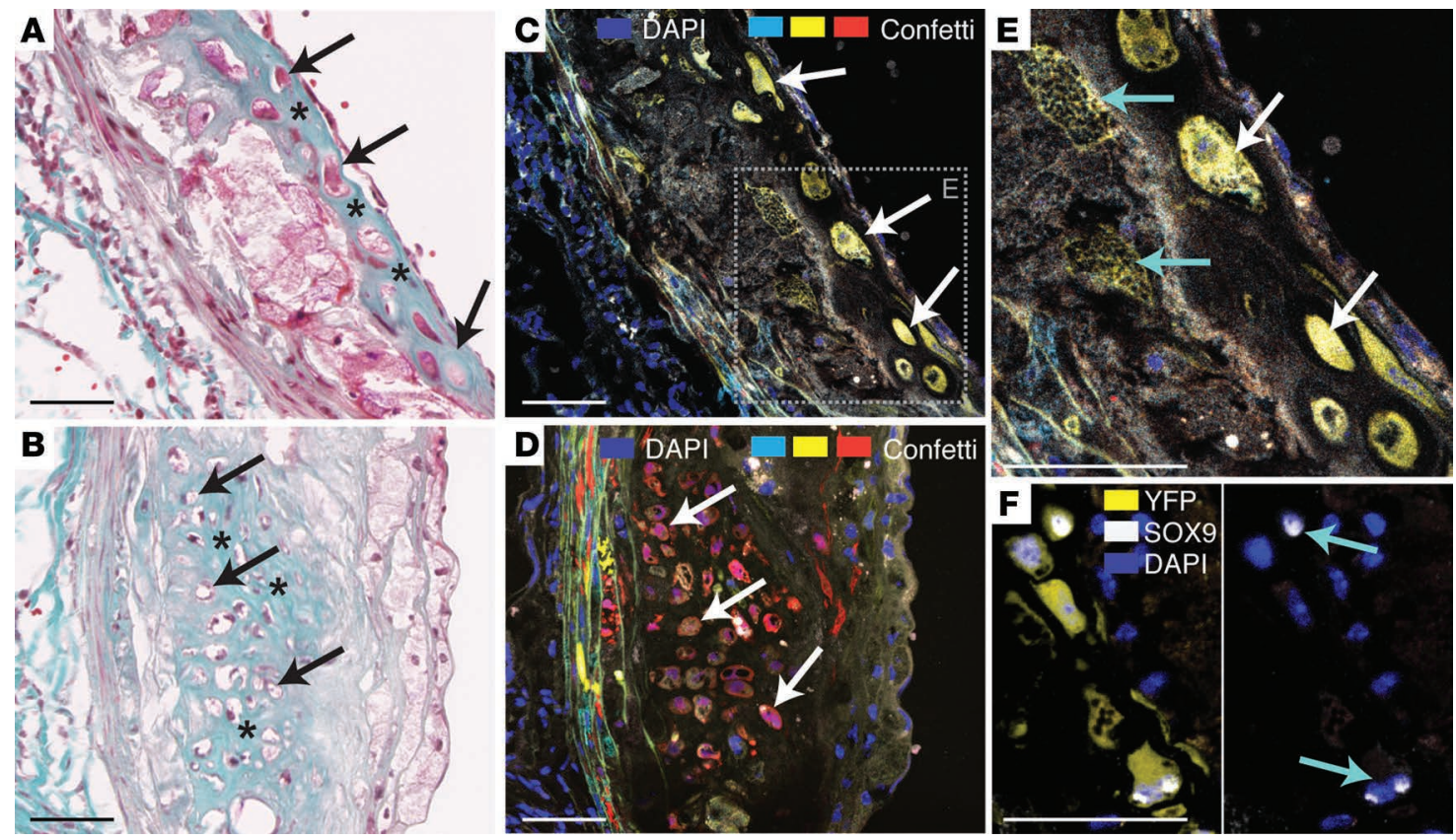

Figure 8. Clonally expanded SMCs underlie plaque chondroid metaplasia. (A and B) Two areas of chondroid metaplasia showing cells with chondrocyte-like morphology (arrows) embedded in a homogeneous matrix staining with the Light Green component of Masson's trichrome stain (asterisks). (C and D) Fluorescence microscopic analysis of an adjacent section shows that the chondrocyte-like cells are expanded from single cells. (E) Larger magnification of inset in C showing patch with several SMC phenotypes. The YFP' patch contains not only chondrocyte-like cells (white arrows), but also underlying SMCs with foamy cytoplasm (cyan arrows). (F) Chondrocyte-like SMCs in an adjacent section to that of $\mathbf{C}$ with nuclear expression of the chondrogenic transcription factor SOX9 (cyan arrows). Scale bars: $50 \mu \mathrm{m}$.

pathophysiological roles in plaque development. This was confirmed by 2 recent studies in which genetic deletion of crucial transcription factors for contractile and modulated SMC phenotypes exerted large effects on plaque progression in $A p o e^{-/-}$mice $(14,27)$. However, the spectrum of SMC phenotypes in atherosclerosis, and the pro- or antiatherogenic effector functions that each of these may have, still needs to be identified.

Here, we find that many modulated SMCs in the plaque interior acquired a characteristic foamy cytoplasm and contained neutral lipids. This is consistent with findings in human atherosclerosis of cells double-positive for lipids and ACTA2 (28). Some of the foamy SMCs also contained reflective material, similar in appearance to cholesterol crystals previously reported in plaque macrophages (29). The functional implications for atherogenesis of lipid accumulation and potential cholesterol crystal formation in modulated SMCs would be relevant to test, and the demonstration of these features in the PCSK9-induced mouse atherosclerosis model facilitates such future studies.

Previous analyses of cultured SMCs as well as plaque SMCs in mouse and human plaques have found that modulated SMCs can acquire a macrophage-like cell phenotype, characterized by the expression of proteins that have traditionally been used to identify plaque macrophages, including LGALS3 and CD68 $(2,14,30)$. We costained for LGALS3/CD68; however, although cells frequently coexisted in the same regions of lesions, we found no examples of cells that were double-positive for LGALS3/CD68 and one of the Confetti colors. The reason for this apparent discrepancy to previous reports may lie in differences in sensitivity or specificity between staining protocols and image analysis procedures. On one hand, some previous reports have used staining procedures that are probably more sensitive than our standard 2-layer fluorescence protocol (2). On the other hand, our multichannel microscopy makes it easy to distinguish autofluorescence from colocalized tracking and marker signals (Supplemental Figure 3), which can otherwise be a specificity concern. Although there is no a priori reason to think that the type of atherosclerosis model is important, it is also worth noting that previous findings of $\mathrm{LGALS}^{+}$and $\mathrm{CD} 68^{+} \mathrm{SMCs}$ in mouse plaques were all made in the Apoe $e^{-/}$model.

Single medial SMCS have diverse phenotypic potential. The SMC patches that we identified often contained several different SMC phenotypes, demonstrating that single medial SMCs possess the potential to modulate 
into several different phenotypes and speaking against the idea that medial SMCs are prespecified toward particular fates. The spatial organization of SMC phenotypes with ACTA2 ${ }^{+}$cells near the endothelium, and foamy and chondrocyte-like SMCs in the plaque interior, indicate that the main controller of SMC phenotypes may be extracellular cues.

Effect of the Confetti transgene on atherosclerosis. The reduction of atherosclerosis in Myh11-CreER ${ }^{T 2}$ Confetti mice compared with Myh11-CreER ${ }^{T 2}$ littermates was unexpected. The 2 groups of mice underwent identical procedures, including tamoxifen injections, pointing directly toward an antiatherogenic role of the Confetti transgene. The Confetti transgene is inserted into the ROSA26 locus and contains neo under the PGK promoter, which - in the absence of recombination - is expressed and blocks transcription of the downstream fluorescent protein genes (15). The antiatherosclerotic effect of the Confetti transgene could therefore be caused by ubiquitous expression of neomycin phosphotransferase, haploinsufficiency of the ROSA26 lncRNAs, the SMC-restricted DNA recombination, or effects of fluorescent protein expression in SMCs. While none of these are obvious candidates for the antiatherosclerotic effect, none of them have, to our knowledge, been directly tested. It would be prudent to clarify this in future studies, since many of these elements are widespread in experimental atherosclerosis research. Although the unexpected effect of the Confetti transgene on atherosclerosis is a limitation of our experiments, the consistency in the results of the aggregation chimeras and Confetti mice argues against a biasing effect on our conclusions on fibrous cap SMC clonality and architecture.

\section{Methods}

Embryo aggregation. Apoe $e^{-/-}$mice (B6.129P2-Apoe $e^{\mathrm{tm} 1 \mathrm{Unc}}$; Taconic M\&B), backcrossed more than 10 times to $\mathrm{B} 6$ mice, and $e G F P^{+} \mathrm{B} 6$ mice (C57BL/6-Tg[CAG-EGFP]1Osb/J; The Jackson Laboratory) were intercrossed to obtain $e G F P^{+} a p o E^{-/-}$mice (hemizygous for the eGFP transgene). Chimeras were created by aggregating 8-cell embryos from matings of $A p o e^{-/-}$and $e G F P^{+} A p o e^{-/-}$mice. Briefly, timed pregnant mice were sacrificed at 2.5 days postcoitum, and zona pellucidae of 8 cell embryos were dissolved in acidic Tyrode's medium (T1788, MilliporeSigma). Two embryos were placed in "well-of-the-wells" depressions created with a blunt needle covered with embryo culture medium under mineral oil, and incubated overnight to allow blastocyst development. Blastocysts were transferred to pseudopregnant mice and allowed to develop to term. Mice were fed normal chow until 10 months of age to allow atherosclerosis to develop.

Multicolor fluorescent transgene experiment. Myh11-CreER ${ }^{T 2}$ mice (B6.FVB-Tg[Myh11-cre/ERT2]1Soff/J, The Jackson Laboratory), expressing tamoxifen-inducible CRE under the SMC-specific Myh11 promoter

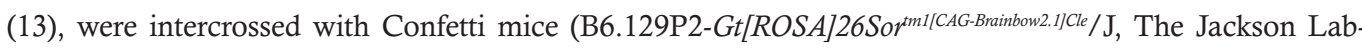
oratory), which harbor a ROSA26 knock-in of an expression cassette consisting of the CAG promoter, a floxed PGK-Neo ${ }^{\mathrm{r}}$-pA cassette serving as a transcriptional roadblock, and the Brainbow 2.1 transgene (15). Both lines were extensively backcrossed into B6 mice. Myh11-CreER ${ }^{T 2}$ Confetti mice (heterozygous for the Confetti knock-in) and Myh11-CreER $R^{\mathrm{T} 2}$ littermates were treated with a 10-day series of once-daily 1 mg i.p. injections of tamoxifen (T5648, MilliporeSigma) dissolved in peanut oil from 6-8 weeks of age. Three to 5 days after the last injection, hypercholesterolemia and atherosclerosis was induced by i.v. injection of rAAV8-mD377Y-mPCSK9 virus particles $\left(1 \times 10^{11}\right.$ vector genomes, produced at Vector Core at the University of North Carolina, Chapel Hill, North Carolina, USA) combined with feeding a high-fat diet (D12079B, Research Diets Inc.) containing $21 \%$ fat and $0.2 \%$ cholesterol as previously described (16). Plasma total cholesterol was measured in duplicate with an enzymatic cholesterol reagent (CHOD-PAP, Roche Diagnostics). Myh11-CreER ${ }^{T 2}$ Confetti mice and Myh11-CreER ${ }^{T 2}$ mice were terminated at 12 weeks $(n=18$ and $n=8$, respectively), 24 weeks ( $n=16$ and $n=10)$, and 36 weeks ( $n=19$ and $n=10)$ after virus injection. Briefly, mice were anesthetized by i.p. injection of pentobarbital $(250 \mathrm{mg} / \mathrm{kg})$ and lidocain $(20 \mathrm{mg} / \mathrm{kg})$, perfused with Cardioplex (30 seconds) and 4\% phosphate-buffered formaldehyde (5 minutes) at $100 \mathrm{mmHg}$ through the left ventricle, and then immersion-fixed in 4\% phosphate-buffered formaldehyde for 6 hours.

Tissue processing and microscopy. The aortic arch and aortic root were cryoprotected in a sucrose solution, embedded in OCT compound, and snap-frozen. Cross-sections of the aortic root were obtained starting from the commissures of the aortic valves until no lesions were present. Serial sections at each approximately $200 \mu \mathrm{m}$ were washed, DAPI-stained, and mounted for microscopy. The aortic arch was cut longitudinally, and sections near the midline were chosen for analysis. Separate sections from the 24- and 36-week time points were stained for LGALS3, CD68, and SOX9 using rat monoclonal anti-LGALS3 (CedarLane 
Laboratories, CL8942), rabbit polyclonal anti-CD68 (Abcam, ab125212), and rabbit polyclonal anti-SOX9 (Abcam, ab185230), followed by Alexa 647-conjugated chicken anti-rat (Molecular Probes, A21472) or goat anti-rabbit (Molecular Probes, A2125) secondary antibodies.

Slides from aggregation chimeras were analyzed with an Olympus Cell-R epifluorescence microscope system equipped with differential interference contrast (DIC) optics. Slides from Myh11-CreER ${ }^{T 2}$ Confetti mice were analyzed using a Nikon A1R scanning confocal system coupled to a Nikon Ti-Eclipse microscope with a Pan Fluor $\times 40 / 1.3$ Oil objective. The entire root was scanned by acquiring multiple images with 3 sequential captures. First, several channels were acquired simultaneously with the $405 \mathrm{~nm}$ (DAPI), $488 \mathrm{~nm}$ nGFP, $562 \mathrm{~nm}$ RFP, and $642 \mathrm{~nm}$ (autofluorescence/LGALS3 stain) lasers. Second, mCFP and YFP channels were acquired with $457 \mathrm{~nm}$ and $514 \mathrm{~nm}$ lasers, respectively. Third, confocal reflection images were obtained to visualize crystals using the 514-nm laser, a beam splitter, and a window of detection of 525-550 nm. All acquisition and postprocessing settings were tested with sections from $M y h 11-C r e E R^{T 2}$ controls to confirm specificity. NIS Elements AR4.30.02 software was used for image acquisition, and ImageJ (NIH) was used for reconstructing total aorta images and to quantify recombination outcomes, plaque volume, number of SMC profiles, and cellularity. Total recombination efficiency and expression of each of the Confetti colors in the arterial media was determined by examining nucleated cells present in the field of view for fluorescence. Plaque volume was calculated by multiplying the average plaque area in serial sections with the total distance of the ascending aorta over which the serial sections were collected. Estimates of the total number of SMCs in plaques were produced by manual counting of fluorescent SMC cellular profiles in plaques (both profiles with and without nucleus present in section). Cellularity of lesions was determined by automatic counting of DAPI-stained nuclei (using a threshold in the DAPI channel followed by particle counting) divided by plaque area.

Staining. Neutral lipids (Oil Red O) and contractile SMCs (ACTA2) were stained in sections at the 24- and 36-week time points after confocal microscopy of the Confetti fluorescent proteins and removal of the coverslip. For ACTA2, we used heat-induced antigen retrieval in citrate buffer (pH 6), rabbit anti-ACTA2 (RB-9010PO, 1:400, Thermo Fisher Scientific), and Flex Envision (Dako). Stained sections were scanned on a digital slide scanner and the images carefully aligned with the earlier obtained confocal microscopy images using Photoshop (Adobe). Mallory's trichrome stain was performed on adjacent sections by standard protocol.

Statistics. Bars in dot blots represent mean \pm SEM. $P$ values were calculated using 2-tailed unpaired Student's $t$ test, one-way ANOVA with Tukey's post-test, or 2-way ANOVA, as indicated. In some cases, data were log-transformed before analysis. Comparison of distributions of medial SMC colors and patch colors were performed with $\chi^{2}$ test. To test whether our data were compatible with the hypothesis that each medial SMC can only produce one plaque SMC phenotype, we calculated the expected number of cases where 2 random fluorescent medial SMCs would produce patches of the same color as $n \sum_{i} x_{i}^{2} /\left(\left[\Sigma_{i} x_{i}^{2}\right]+\Sigma_{i \neq j} x_{i} x_{j}\right)$ and those where 2 random fluorescent medial SMCs would produce patches of different colors as $n\left(\Sigma_{i \neq j} x_{i} x_{j}\right) /$ $\left(\left[\Sigma_{i} x_{i}^{2}\right]+\Sigma_{i \neq j} x_{i} x_{j}\right)$, with $n$ being the number of observations and $x_{i}$ being the fraction of fluorescent medial SMCs with color $i \in$ mCFP, nGFP, YFP, RFP . The observed ratio was subsequently compared with the expected ratio using a 2-tailed binomial test. $P<0.05$ was considered statistically significant. All statistical tests were performed in Prism 6 (GraphPad Software).

Study approval. The Danish Animal Experiments Inspectorate approved animal procedures, which were all conducted at Aarhus University (approval no. 2012-15-2934-00286).

\section{Author contributions}

KJ, MBL, JS, EMF, MK, and JFB designed experiments; KJ, MBL, JS, and SG conducted experiments; $\mathrm{KJ}, \mathrm{MBL}, \mathrm{MK}$, and LC acquired data; KJ, MBL, LC, and JFB drafted the manuscript; and all authors contributed critically to revision of manuscript.

\section{Acknowledgments}

The study was funded by grants from the Danish Independent Research Council (Sapere Aude Programme, 4004-00459B) and the Ministerio de Economia, Industria e Competividad (MEIC) with cofunding from the Fondo Europeo de Desarrollo Regional (FEDER) (SAF2016-75580-R) and by scholarships from the Danish Independent Research Council (to MBL) and the Novo Scholarship Program (to KJ). The CNIC is supported by MEIC and the Pro CNIC Foundation, and is a Severo Ochoa Center of Excellence (SEV-2015-0505). We would like to thank Leticía González, Lisa Maria Røge, and Dorte Wilhart Qualmann for histology and genotyping; Lisbeth Ahm Hansen and Peter Kragh for embryo work; the CNIC microscopy Unit (Veronica 
Labrador, Elvira Arza, and Antonio Santos-Beneit) for support on confocal microscopy; and the CNIC Histopathology Unit (Roisin Doohan, Brenda Guijarro, and Antonio Molina) for histological stainings and tissue scans.

Address correspondence to: Jacob F. Bentzon, Experimental Pathology of Atherosclerosis, Centro Nacional de Investigaciones Cardiovasculares Carlos III (CNIC), Melchor Fernández Almagro 3, 28029 Madrid, Spain. Phone: 34.914531200, ext. 2012; Email: jfbentzon@cnic.es.

1. Gomez D, Shankman LS, Nguyen AT, Owens GK. Detection of histone modifications at specific gene loci in single cells in histological sections. Nat Methods. 2013;10(2):171-177.

2. Feil S, et al. Transdifferentiation of vascular smooth muscle cells to macrophage-like cells during atherogenesis. Circ Res. 2014;115(7):662-667.

3. Bentzon JF, Weile C, Sondergaard CS, Hindkjaer J, Kassem M, Falk E. Smooth muscle cells in atherosclerosis originate from the local vessel wall and not circulating progenitor cells in ApoE knockout mice. Arterioscler Thromb Vasc Biol. 2006;26(12):2696-2702.

4. Falk E, Nakano M, Bentzon JF, Finn AV, Virmani R. Update on acute coronary syndromes: the pathologists' view. Eur Heart J. 2013;34(10):719-728

5. Kolodgie FD, et al. The thin-cap fibroatheroma: a type of vulnerable plaque: the major precursor lesion to acute coronary syndromes. Curr Opin Cardiol. 2001;16(5):285-292.

6. Matthews C, et al. Vascular smooth muscle cells undergo telomere-based senescence in human atherosclerosis: effects of telomerase and oxidative stress. Circ Res. 2006;99(2):156-164.

7. Wang J, et al. Vascular Smooth Muscle Cell Senescence Promotes Atherosclerosis and Features of Plaque Vulnerability. Circulation. 2015;132(20):1909-1919.

8. Lutgens E, de Muinck ED, Kitslaar PJ, Tordoir JH, Wellens HJ, Daemen MJ. Biphasic pattern of cell turnover characterizes the progression from fatty streaks to ruptured human atherosclerotic plaques. Cardiovasc Res. 1999;41(2):473-479.

9. Murry CE, Gipaya CT, Bartosek T, Benditt EP, Schwartz SM. Monoclonality of smooth muscle cells in human atherosclerosis. Am J Pathol. 1997;151(3):697-705.

10. Chung IM, Schwartz SM, Murry CE. Clonal architecture of normal and atherosclerotic aorta: implications for atherogenesis and vascular development. Am J Pathol. 1998;152(4):913-923.

11. Greif DM, et al. Radial construction of an arterial wall. Dev Cell. 2012;23(3):482-493.

12. Getz GS, Reardon CA. Apoprotein E as a lipid transport and signaling protein in the blood, liver, and artery wall. J Lipid Res. 2009;50(suppl):S156-S161.

13. Wirth A, et al. G12-G13-LARG-mediated signaling in vascular smooth muscle is required for salt-induced hypertension. Nat Med. 2008;14(1):64-68.

14. Shankman LS, et al. KLF4-dependent phenotypic modulation of smooth muscle cells has a key role in atherosclerotic plaque pathogenesis. Nat Med. 2015;21(6):628-637.

15. Snippert HJ, et al. Intestinal crypt homeostasis results from neutral competition between symmetrically dividing Lgr5 stem cells. Cell. 2010;143(1):134-144

16. Bjørklund MM, et al. Induction of atherosclerosis in mice and hamsters without germline genetic engineering. Circ Res. 2014;114(11):1684-1689.

17. Naik V, et al. Sources of cells that contribute to atherosclerotic intimal calcification: an in vivo genetic fate mapping study. Cardiovasc Res. 2012;94(3):545-554.

18. Hoglund VJ, Majesky MW. Patterning the artery wall by lateral induction of Notch signaling. Circulation. 2012;125(2):212-215 .

19. Stary HC, et al. A definition of the intima of human arteries and of its atherosclerosis-prone regions. A report from the Committee on Vascular Lesions of the Council on Arteriosclerosis, American Heart Association. Arterioscler Thromb. 1992;12(1):120-134

20. Gardner SE, Humphry M, Bennett MR, Clarke MC. Senescent vascular smooth muscle cells drive inflammation through an interleukin-1 $\alpha$-dependent senescence-associated secretory phenotype. Arterioscler Thromb Vasc Biol. 2015;35(9):1963-1974.

21. Clowes AW, Schwartz SM. Significance of quiescent smooth muscle migration in the injured rat carotid artery. Circ Res. 1985;56(1):139-145.

22. Chappell J, et al. Extensive proliferation of a subset of differentiated, yet plastic, medial vascular smooth muscle cells contributes to neointimal formation in mouse injury and atherosclerosis models. Circ Res. 2016;119(12):1313-1323.

23. Pontes-Quero S, et al. Dual ifgMosaic: a versatile method for multispectral and combinatorial mosaic gene-function analysis Cell. 2017;170(4):800-814.e18

24. Bray SJ. Notch signalling in context. Nat Rev Mol Cell Biol. 2016;17(11):722-735.

25. Manderfield LJ, et al. Notch activation of Jagged1 contributes to the assembly of the arterial wall. Circulation. 2012;125(2):314-323.

26. Lindner V, Booth C, Prudovsky I, Small D, Maciag T, Liaw L. Members of the Jagged/Notch gene families are expressed in injured arteries and regulate cell phenotype via alterations in cell matrix and cell-cell interaction. Am J Pathol. 2001;159(3):875-883.

27. Ackers-Johnson M, et al. Myocardin regulates vascular smooth muscle cell inflammatory activation and disease. Arterioscler Thromb Vasc Biol. 2015;35(4):817-828.

28. Allahverdian S, Chehroudi AC, McManus BM, Abraham T, Francis GA. Contribution of intimal smooth muscle cells to cholesterol accumulation and macrophage-like cells in human atherosclerosis. Circulation. 2014;129(15):1551-1559.

29. Duewell P, et al. NLRP3 inflammasomes are required for atherogenesis and activated by cholesterol crystals. Nature. 2010;464(7293):1357-1361.

30. Vengrenyuk Y, et al. Cholesterol loading reprograms the microRNA-143/145-myocardin axis to convert aortic smooth muscle cells to a dysfunctional macrophage-like phenotype. Arterioscler Thromb Vasc Biol. 2015;35(3):535-546. 